\title{
EQUILIBRIUM VORTEX CONFIGURATIONS IN DOMAINS WITH BOUNDARY
}

\author{
BY \\ KENNETH G. MILLER \\ Department of Mathematics, Wichita State University, Wichita, Kansas
}

\begin{abstract}
Given a stable configuration of point vortices for steady two-dimensional inviscid, incompressible fluid flow in a domain $D$, it is shown that there is another stable configuration of stationary vortices in $D$ with vortices near the original vortices plus additional vortices near any points on the boundary where the speed of the original flow is a nonzero relative minimum.
\end{abstract}

1. Introduction. The aim of this paper is to prove the following result, which can be used to determine stable equilibrium configurations of point vortices in a two-dimensional domain $D$ with boundaries:

Given a two-dimensional steady incompressible fluid flow that is irrotational except for possibly a finite number of stable point vortices, let $q$ be the restriction of the fluid speed to the boundary $\Gamma$ of the flow domain $D$. Suppose there are points $P_{1}, P_{2}, \ldots, P_{n}$ on $\Gamma$ such that $q$ has a nondegenerate relative minimum at each $P_{j}, P_{j}$ not a stagnation point of the flow. Then there exist steady flows in $D$ with point vortices near each of the original vortices plus additional stable vortices near each of the points $P_{j}$.

By Bernoulli's Theorem the result states that there is a perturbed flow with an additional stable stationary vortex near any non-stagnation point on the boundary where the pressure of the original flow is a relative maximum. The orientation of an added vortex is determined by the direction of the original flow near $P_{j}$ : If $P_{j}$ is to the right (respectively, left) of fluid particles flowing past $P_{j}$, then a clockwise (resp., counter-clockwise) vortex can be added near $P_{j}$.

The term stagnation point is used in the following restricted sense: $P \in \Gamma$ is a stagnation point if $q=0$ at $P$ and $P$ is a branch point for the flow, i.e., an interior streamline has an endpoint at $P$. If the boundary is smooth (analytic) at $P$, then $P$ is a stagnation point if and only if $q=0$ at $P$. However, if $P$ is a convex corner of the boundary (interior angle $<\pi$ ), then $q$ is necessarily 0 at $P$, but $P$ is generally not a stagnation point. According to the statement above, if $P$ is a convex corner of the flow domain and not a

Received February 14, 1996.

1991 Mathematics Subject Classification. Primary 76C.

(C)1998 Brown University 
stagnation point, then it is possible to add a stable vortex near $P$. This was proved in [7]. In the present paper we therefore restrict attention to the case of smooth boundary points.

In considering the possible configuration of vorticity in the steady state for a twodimensional incompressible fluid, point vortex configurations provide important information. It was shown by Berger and Fraenkel [1] and Turkington [10] that if the vorticity is sufficiently concentrated, then the flow is a desingularization of a point vortex flow. Conversely, any configuration of point vortices in stable equilibrium can be desingularized ([11], [12], [3]).

Nevertheless, configurations of point vortices in equilibrium with respect to a fixed boundary have not been studied extensively. Saffman and Sheffield [9] and Huang and Chow [5] have considered a single vortex in equilibrium with respect to an airfoil. Section $3 \mathrm{c}$ below briefly discusses these vortices in the context of the present paper. Examples of stable corner vortex configurations are given in [7]. Several more examples based on the result stated above are given in Section 3 below. It is well known (see [4]) that there are flows with one stationary vortex in a bounded simply connected domain; in Section 3a it is shown that generally there are flows with two stationary stable vortices in a bounded simply connected domain. In Section $3 \mathrm{~b}$ it is shown that in an annular domain there are steady flows with at least two stable vortices. In Section $3 \mathrm{~d}$ it is shown that for an airfoil at a positive angle of attack above a flat ground, there can be a stable vortex attached to the ground.

As noted in [7], in the case of a corner the result can be applied successively to obtain flows with a finite sequence of arbitrary length of alternately positive and negative vortices descending into any convex corner of $D$. This is not possible at smooth boundary points. Generally, if a vortex is added near a smooth boundary point $P$ where the speed of the original flow is a minimum, then the new flow will have two stagnation points, one on either side of $P$, and the speed of the new flow will have a local maximum, but no minimum, between the stagnation points. (However, it is possible to add a secondary vortex near a sharply rounded corner.) Consequently, there seems to be an upper bound, depending on the geometry of $D$, on the number of vortices in stable equilibrium in a domain with no convex corners, that upper bound being small for simple geometries. (Even in the case of a sequence of vortices in a convex corner, the number of vortices that are physically significant is small since the strengths of additional vortices must decrease rapidly.) That the number of possible vortices in a stable vortex configuration is small is related to phenomena that appear in the study of turbulent flow. In numerical simulations of two-dimensional turbulence, systems of concentrated vortices are observed to emerge from a generic or random initial vorticity distribution after some evolution. As time progresses, some of the vortices intermingle and eventually merge together, resulting in just a few large-scale vortices as the quasi-steady state.

The main result is stated more precisely and proved in the next section. Several situations in which it can be applied are described in Section 3.

2. Existence of stable vortex configurations. Let $D$ be a connected domain in the plane with a boundary consisting of finitely many non-intersecting simple piecewise 
analytic curves $\Gamma_{j}$. It is assumed that each $\Gamma_{j}$ is either closed or extends to infinity in both directions. It is also assumed that if $\Gamma_{j}$ is not analytic at $P$ then it has a corner at $P$. $D$ is conformally equivalent to a canonical domain $\widetilde{D}$ where canonical domains are taken to be slit domains that have a boundary consisting of the real axis $\Gamma$ and finitely many line segments in the upper half-plane parallel to $\Gamma$. Let $\phi_{0}$ be the complex potential for an incompressible flow in $D$ that is irrotational except possibly at a finite number of point vortices. Let $P$ be a boundary point of $D$, let $f: \widetilde{D} \rightarrow D$ be conformal, with $f(0)=P, f^{\prime}(0) \neq 0$, and let $\phi=\phi_{0} \circ f$. (Although $\phi$ may be multiply-valued in the multiply-connected case, $\phi^{\prime}$ is a single-valued analytic function.) The following lemma states the crucial hypothesis of the main theorem in analytic terms.

LEMMA 1. Let $q$ be the fluid speed considered as a function restricted to the boundary of $D$. Then $q$ has a nondegenerate local minimum at a smooth boundary point $P=f(0)$, with $q(P) \neq 0$, if and only if $\phi^{\prime}(0) \neq 0$,

$$
\operatorname{Re}\left(\frac{\phi^{\prime \prime}}{\phi^{\prime}}-\frac{f^{\prime \prime}}{f^{\prime}}\right)(0)=0
$$

and

$$
\operatorname{Re}\left(\frac{\phi^{\prime \prime}}{\phi^{\prime}}-\frac{f^{\prime \prime}}{f^{\prime}}\right)^{\prime}(0)>0 .
$$

Proof. The fluid velocity $v$ at $\zeta=f(z)$ considered as a function on $\widetilde{D}$ is given by $\bar{v}=\phi^{\prime}(z) / f^{\prime}(z)$. Therefore,

$$
2|v| \frac{\partial|v|}{\partial z}=\frac{\partial|v|^{2}}{\partial z}=v \frac{\partial \bar{v}}{\partial z}=v \bar{v}\left(\frac{\phi^{\prime \prime}}{\phi^{\prime}}-\frac{f^{\prime \prime}}{f^{\prime}}\right) .
$$

(The notation $\frac{\partial}{\partial z}=\frac{1}{2}\left(\frac{\partial}{\partial x}-i \frac{\partial}{\partial y}\right)$ and $\frac{\partial}{\partial \bar{z}}=\frac{1}{2}\left(\frac{\partial}{\partial x}+i \frac{\partial}{\partial y}\right)$ for derivatives of functions of a complex variable $z=x+i y$ is used throughout.) Consequently,

$$
\frac{\partial q}{\partial z}=q w
$$

where $w=\frac{1}{2}\left(\left(\phi^{\prime \prime} / \phi^{\prime}\right)-\left(f^{\prime \prime} / f^{\prime}\right)\right)$. Since $\partial q / \partial x=2 \operatorname{Re}(\partial q / \partial z)$, it follows that (1) is equivalent to $q$ (transplanted to the real axis) having a critical point at 0 . Also $\partial^{2} q / \partial z^{2}=$ $\left(\partial w / \partial z+w^{2}\right) q$ and $\partial^{2} q / \partial z \partial \bar{z}=w \partial q / \partial \bar{z}=q \bar{w} w$ imply that

$$
\frac{\partial^{2} q}{\partial x^{2}}=2 \operatorname{Re}\left(\frac{\partial^{2} q}{\partial z^{2}}+\frac{\partial^{2} q}{\partial z \partial \bar{z}}\right)=q\left(2 \operatorname{Re} \frac{\partial w}{\partial z}+4(\operatorname{Re} w)^{2}\right) .
$$

Since (1) holds at a critical point, it follows that (2) is the condition that the transplanted function $q$ have a nondegenerate local minimum at 0 .

By the Kirchhoff-Routh theory (see Lin [6] or Saffman [8]) there is a function $W\left(\zeta_{1}, \ldots\right.$, $\left.\zeta_{p}\right)$, the Routh function, which is a Hamiltonian for the time-dependent motion of $p$ point vortices located at $\left(\zeta_{1}, \ldots, \zeta_{p}\right)$ with strengths $k_{1}, \ldots, k_{p}$. The flow is steady if the vortices are located at a critical point $\left(\widetilde{\zeta}_{1}, \ldots, \widetilde{\zeta}_{p}\right)$ of $W$ and is stable in the Hamiltonian sense if that critical point is a local minimum of $W$. We consider only configurations of stationary vortices for which the second derivative matrix satisfies the nondegeneracy condition

$$
D^{2} W\left(\widetilde{\zeta}_{1}, \ldots, \widetilde{\zeta}_{p}\right)>0
$$


The function $W$ is given explicitly by

$$
W\left(\zeta_{1}, \ldots, \zeta_{p}\right)=\sum_{j=1}^{p}\left(-k_{j} \eta\left(\zeta_{j}\right)+k_{j}^{2} H\left(\zeta_{j}\right)\right)-\frac{1}{2} \sum_{j=1}^{p} \sum_{m \neq j} k_{j} k_{m} g\left(\zeta_{j}, \zeta_{m}\right)
$$

where $g(z, w)=-\frac{1}{2 \pi} \log |z-w|-h(z, w)$ is a Green's function which, for every $w \in D$, is constant on each component of $\partial D$, the constants being chosen so that $\int_{\Gamma_{j}} \partial g / \partial n d s=0$ on each inner boundary; $H(\zeta)=\frac{1}{2} h(\zeta, \zeta)$; and $\eta$ is a harmonic function on $D$ that is constant on each of the components of $\partial D$. The function $\eta$ can be chosen so that the flow has prescribed circulations around each of the inner boundaries of $D$ and prescribed velocity at infinity if $D$ is unbounded.

The stream function for the flow is given by

$$
\psi(\zeta)=\eta(\zeta)+\sum_{m=1}^{p} k_{m} g\left(\zeta, \zeta_{m}\right)
$$

Let $\psi_{\nu}(P)=\frac{\partial \psi}{\partial \nu}(P)$ denote the directional derivative in the direction of the exterior normal at points $P$ on the boundary. The sign of $\psi_{\nu}(P)$ determines the direction of the flow near $P: \psi_{\nu}(P)>0$ if $P$ is to the left of fluid particles flowing past $P$. Note that if $f: \widetilde{D} \rightarrow D$ is conformal, $f(0)=P, f^{\prime}(0) \neq 0, \phi=\phi_{0} \circ f$, with $\widetilde{D}$ and $\phi_{0}$ as above, then $\phi^{\prime}(0)$ is real and

$$
\operatorname{sgn} \psi_{\nu}(P)=-\operatorname{sgn} \phi^{\prime}(0) .
$$

The following is the main result of the paper.

THEOREM 2. Let $\widetilde{\zeta}_{1}, \ldots, \widetilde{\zeta}_{p}, p \geq 0$, be a stationary configuration of point vortices in $D$ with strengths $\tilde{k}_{1}, \ldots, \tilde{k}_{p}$, respectively, for which (3) is satisfied, and let $\psi$ be the corresponding stream function. Let $P_{1}, \ldots, P_{n}$ be distinct boundary points, none a stagnation point of the flow, such that the speed $q=\left|\psi_{\nu}\right|$, when restricted to the boundary, has a nondegenerate local minimum at each $P_{j}$. Let $\delta_{j}=\operatorname{sgn} \psi_{\nu}\left(P_{j}\right)$. Then for any $k=\left(k_{1}, \ldots, k_{n}\right)$ with $k_{j}>0$ sufficiently small, there exists a configuration $\zeta_{1}^{\prime}, \ldots, \zeta_{n}^{\prime}$, $\zeta_{1}, \ldots, \zeta_{p}$ of $n+p$ stationary vortices in $D$, for the same potential function $\eta$, with strengths $\delta_{1} k_{1}, \ldots, \delta_{n} k_{n}, \tilde{k}_{1}, \ldots, \tilde{k}_{p}$ respectively, where

$$
\lim _{k \rightarrow 0} \zeta_{j}^{\prime}(k)=P_{j} \text { for } 1 \leq j \leq n \text { and } \lim _{k \rightarrow 0} \zeta_{j}(k)=\widetilde{\zeta}_{j} \text { for } 1 \leq j \leq p .
$$

The new configuration is stable in the sense that it also satisfies the nondegeneracy condition (3).

It suffices to prove the theorem with $n=1$, since if $n-1$ vortices $\zeta_{1}^{\prime}(k), \ldots, \zeta_{n-1}^{\prime}(k)$ can be added, then for $k_{1}, \ldots, k_{n-1}$ sufficiently small, the speed for the resulting flow will have a nondegenerate local minimum at some boundary point near $P_{n}$, and the result with $n=1$ can be applied to add a vortex near $P_{n}$. With $n=1$ the subscripts on $P, \delta$, and $k$ will be dropped. Since the case when $P$ is a corner was proved in [7], we may assume that the boundary is analytic at $P$. (Also the case when $P$ is a corner follows from the smooth case by a limiting argument.)

For the variable $\zeta^{\prime}$ corresponding to the new vortex we will use a conformal map $f$ : $\widetilde{D} \rightarrow D$ from a canonical domain as described above onto $D$ with $f(0)=P$. Denoting 
the Routh function for $p+1$ vortices in $D$ with strengths $\delta k, \tilde{k}_{1}, \ldots, \tilde{k}_{p}$ by $\widetilde{W}$, we consider the function

$$
W(z, \zeta)=\widetilde{W}\left(f(z), \zeta_{1}, \ldots, \zeta_{p}\right)
$$

There are two parts to the proof of the theorem. First, to establish the existence of equilibrium configurations the implicit function theorem will be used to show that the system

$$
\frac{\partial W}{\partial z}(z, \zeta, k)=0, \quad \frac{\partial W}{\partial \zeta_{j}}(z, \zeta, k)=0, \quad 1 \leq j \leq p,
$$

can be solved for $(z, \zeta)$ in terms of $k$ for $k$ positive and near 0 . Later, an analysis of the eigenvalues of the second derivative matrix will be used to show that the nondegeneracy condition (3) is satisfied.

Let $W_{0}$ be the Routh function for $p$ vortices on $D$. Then

$$
\frac{\partial W}{\partial \zeta_{j}}(z, \zeta)=\frac{\partial W_{0}}{\partial \zeta_{j}}(\zeta)-\tilde{k}_{j} \delta k \frac{\partial g}{\partial \zeta_{j}}\left(\zeta_{j}, f(z)\right), \quad 1 \leq j \leq p .
$$

In particular, the $2 p \times 2 p$ block of the Jacobian matrix of the system (7) corresponding to the variables $\zeta=\left(\zeta_{1}, \ldots, \zeta_{p}\right)$ is nonsingular at $k=0, \zeta=\widetilde{\zeta}$ since the original configuration satisfies (3).

The first equation in (7) requires a considerably more detailed analysis. Let $\Omega_{0}$ be the intersection of a neighborhood of $0 \in C$ and the upper half plane, and let $\Omega_{j}, 1 \leq j \leq p$, be neighborhoods of $\widetilde{\zeta}_{j}$ in $D$. We assume that $\Omega_{j}$ and $\Omega_{0}$ are small enough that the sets $f\left(\Omega_{0}\right), \Omega_{1}, \ldots, \Omega_{p}$ are pairwise disjoint. Let $\Omega=\Omega_{1} \times \cdots \times \Omega_{p}$. For $z \in \Omega_{0}$ and $\zeta \in \Omega$ define

$$
\psi_{1}(z, \zeta)=\eta(f(z))+\sum_{m=1}^{p} \tilde{k}_{m} g\left(f(z), \zeta_{m}\right)
$$

By (5), as a function of $z, \psi_{1}(z, \zeta)$ is the stream function for the flow in $D$ (transplanted to $\widetilde{D}$ ) with vortices perturbed from the original stationary configuration $\widetilde{\zeta}$. For each $\zeta \in \Omega$ let $\phi_{1}$ be the corresponding complex potential, i.e., an analytic function of $z$ such that $\psi_{1}(z, \zeta)=\operatorname{Im} \phi_{1}(z, \zeta)$. Then

$$
\frac{\partial W}{\partial z}(z, \zeta)=\frac{i}{2} \delta k \phi_{1}^{\prime}(z, \zeta)+k^{2} \frac{\partial H_{1}}{\partial \zeta}(z)
$$

where $H_{1}(z)=H(f(z))$. $\phi_{1}^{\prime}, \ldots, \phi_{1}^{(k)}$ denote derivatives of $\phi_{1}$ with respect to the complex variable $z$.

We recall that by the Kirchhoff-Routh theory (see Lin [6] or Saffman [8])

$$
H_{1}(z)=\tilde{H}(z)-\frac{1}{4 \pi} \log \left|f^{\prime}(z)\right|,
$$

where $\widetilde{H}(z)=\frac{1}{2} \tilde{h}(z, z)$ and $\tilde{g}(z, w)=-\frac{1}{2 \pi} \log |z-w|-\tilde{h}(z, w)$ is the Green's function for $\widetilde{D}$. It was shown in [7] that

$$
4 \pi \widetilde{H}(z)=-\log |z-\bar{z}|+R(z)
$$


where $R$ is real analytic near the real axis with $R, \partial R / \partial z$, and $\partial R / \partial \bar{z}$ all vanishing on the real axis. Hence

$$
4 \pi \frac{\partial H_{1}}{\partial z}=-\frac{1}{z-\bar{z}}-\frac{1}{2} \frac{f^{\prime \prime}(z)}{f^{\prime}(z)}+\frac{\partial R}{\partial z}
$$

where $\partial R / \partial z=O(\operatorname{Im} z)$.

We can now see problems with the first equation in the system (7). For one, (10) shows that there is a degeneracy when $k=0$. (Simply dividing by $k$ yields the equation

$$
\frac{i}{2} \delta \phi_{1}^{\prime}(z, \zeta)+k \frac{\partial H_{1}}{\partial \zeta}(z)=0
$$

but this equation has no solution for $k=0, \zeta=\tilde{\zeta}$, and $z$ near 0 , since $P$ is not a stagnation point.) Moreover, there is a singularity as $z$ approaches the real axis because of the first term in (13). We will see shortly that these problems can be handled by dividing the first equation in (7) by $k^{2}$, multiplying by $z$ and scaling the variable $\operatorname{Im}(z)$. We note that if $\operatorname{Im} z \neq 0$, then $\partial W / \partial z=0$ if and only if

$$
\operatorname{Re} \frac{\partial W}{\partial z}=0 \text { and } \operatorname{Re}\left(z \frac{\partial W}{\partial z}\right)=0 .
$$

Since $\operatorname{Im} \phi_{1}(z, \zeta)$ is constant for $z$ on the real axis, it follows that

$$
\operatorname{Im} \phi_{1}^{(k)}(z, \zeta)=0 \text { for } k \geq 1 \text { and } z \text { real. }
$$

Therefore with $z=x+i y$, we have the following Taylor expansions:

$$
\begin{gathered}
\operatorname{Im}\left(\phi_{1}^{\prime}(z, \zeta)\right)=y\left[\phi_{1}^{\prime \prime}(0, \zeta)+x \phi_{1}^{\prime \prime \prime}(0, \zeta)+O\left(z^{2}\right)\right] \\
\operatorname{Im}\left(z \phi_{1}^{\prime}(z, \zeta)\right)=y\left[\phi_{1}^{\prime}(0, \zeta)+2 x \phi_{1}^{\prime \prime}(0, \zeta)+O\left(z^{2}\right)\right]
\end{gathered}
$$

From $(10),(13),(15)-(16)$, and the identity $\operatorname{Re} \frac{2 z}{z-\bar{z}}=1$, it follows that

$$
\begin{aligned}
& \frac{-8 \pi}{k^{2}} \operatorname{Re} \frac{\partial W}{\partial z}=4 \pi \delta \frac{y}{k}\left(\phi_{1}^{\prime \prime}(0, \zeta)+x \phi_{1}^{\prime \prime \prime}(0, \zeta)\right)+\operatorname{Re}\left(\frac{f^{\prime \prime}}{f^{\prime}}\right)(0)+x \operatorname{Re}\left(\frac{f^{\prime \prime}}{f^{\prime}}\right)^{\prime}(0)+R_{1} \\
& \frac{-8 \pi}{k^{2}} \operatorname{Re}\left(z \frac{\partial W}{\partial z}\right)=4 \pi \delta \frac{y}{k}\left(\phi_{1}^{\prime}(0, \zeta)+2 x \phi_{1}^{\prime \prime}(0, \zeta)\right)+1+x \operatorname{Re}\left(\frac{f^{\prime \prime}}{f^{\prime}}\right)(0)+R_{2}
\end{aligned}
$$

where the remainder terms $R_{1}$ and $R_{2}$ are both $O(y)+O\left(x^{2}\right)$.

We now introduce the variable $u=y / k$ and let $F(x, u, \zeta, k)$ and $G(x, u, \zeta, k)$ be the analytic functions given by (17) and (18) respectively when $y / k$ is replaced by $u$. Let $\tilde{u}=-\delta /\left(4 \pi \phi^{\prime}(0)\right)$, where $\phi(z)=\phi_{1}(z, \widetilde{\zeta}), \tilde{\zeta}$ the given minimizer of $W_{0}$. It follows from (6) that $\tilde{u}>0$, and from (14) and (1) that

$$
\frac{\phi^{\prime \prime}(0)}{\phi^{\prime}(0)}=\operatorname{Re}\left(\frac{f^{\prime \prime}}{f^{\prime}}\right)(0) \text {. }
$$

Therefore, $F(0, \tilde{u}, \widetilde{\zeta}, 0)=G(0, \tilde{u}, \widetilde{\zeta}, 0)=0$. Also, after some simplification using (19) the Jacobian determinant

$$
\frac{\partial(F, G)}{\partial(x, u)}=\left|\begin{array}{ll}
{\left[4 \pi \delta \phi^{\prime \prime \prime}(0) \tilde{u}+\operatorname{Re}\left(f^{\prime \prime} / f^{\prime}\right)^{\prime}(0)\right]} & 4 \pi \delta \phi^{\prime \prime}(0) \\
{\left[8 \pi \delta \phi^{\prime \prime}(0) \tilde{u}+\operatorname{Re}\left(f^{\prime \prime} / f^{\prime}\right)(0)\right]} & 4 \pi \delta \phi^{\prime}(0)
\end{array}\right|
$$


at $(x, u, \zeta, k)=(0, \tilde{u}, \widetilde{\zeta}, 0)$ is seen to be

$$
4 \pi \delta \phi^{\prime}(0)\left[\operatorname{Re}\left(f^{\prime \prime} / f^{\prime}\right)^{\prime}(0)-\left(\phi^{\prime \prime} / \phi^{\prime}\right)^{\prime}(0)\right],
$$

which is nonzero by (2).

As noted after (8), the Jacobian of the system $\partial W / \partial \zeta=0$ is nonzero for $k=0, \zeta=\widetilde{\zeta}$. Also by (8) the $2 p \times 2$ block of cross terms $\partial^{2} W / \partial x \partial \zeta_{j}$ and $\partial^{2} W / \partial u \partial \zeta_{j}$ vanishes when $(x, u, \zeta, k)=(0, \tilde{u}, \tilde{\zeta}, 0)$. Therefore the Jacobian of the $2 p+2$ system

$$
F(x, u, \zeta, k)=0, \quad G(x, u, \zeta, k)=0, \quad \frac{\partial W}{\partial \zeta_{j}}=0, \quad j=1, \ldots, p
$$

is nonsingular at $(x, u, \zeta, k)=(0, \tilde{u}, \widetilde{\zeta}, 0)$. It follows from the implicit function theorem that for some $\bar{k}>0$ there are functions $x(k), u(k)$, and $\zeta(k)$ defined and analytic for $0<k \leq \bar{k}$ and solving (21). Consequently for each $k, 0<k \leq \bar{k}$, there is a critical point $(z(k), \zeta(k))$ of the Routh function $W, z(k)=x(k)+i y(k)$, satisfying

$$
x(k)=O(k), \quad y(k)=\frac{-\delta k}{4 \pi \phi^{\prime}(0)}+O\left(k^{2}\right)>0, \quad \zeta(k)=\widetilde{\zeta}+O(k) .
$$

It remains to be shown that if $k$ is sufficiently small, then the critical point $(z(k), \zeta(k))$ satisfies the nondegeneracy condition (3) and is therefore a local minimum for $W$.

LEMMA 3. Let $(z, \zeta)=(x(k)+i y(k), \zeta(k))$ be a critical point of $W$ satisfying (22). Then the eigenvalues of the $2 \times 2$ matrix

$$
M=\left[\begin{array}{ll}
W_{x x} & W_{x y} \\
W_{x y} & W_{y y}
\end{array}\right]
$$

have the form

$$
\lambda_{1}=\alpha+O(k) \text { and } \quad \lambda_{2}=\gamma k^{2}+O\left(k^{3}\right)
$$

with $\alpha>0$ and $\gamma>0$ independent of $k$.

Proof. Let $a=2 W_{z \bar{z}}$ and $b=2\left(W_{z \bar{z}}+W_{z z}\right)$. Then

$$
M=\left[\begin{array}{cc}
\operatorname{Re} b & -\operatorname{Im} b \\
-\operatorname{Im} b & 2 a-\operatorname{Re} b
\end{array}\right]
$$

and has eigenvalues

$$
\lambda_{1}=(a+|a-b|) \quad \text { and } \quad \lambda_{2}=(a-|a-b|) .
$$

By (10) and (13),

$$
a=-\frac{k^{2}}{2 \pi}(z-\bar{z})^{-2}+\frac{k^{2}}{2 \pi} \frac{\partial^{2} R}{\partial z \partial \bar{z}}(z)
$$

and

$$
b=i k \delta \phi^{\prime \prime}(z, \zeta)-\frac{k^{2}}{4 \pi}\left[\left(\frac{f^{\prime \prime}}{f^{\prime}}\right)^{\prime}(z)\right]+\frac{k^{2}}{2 \pi}\left(\frac{\partial^{2} R}{\partial z \partial z}(z)+\frac{\partial^{2} R}{\partial z \partial \bar{z}}(z)\right) .
$$

Since $\frac{\partial R}{\partial z}(z)$ vanishes on the $x$-axis,

$$
\frac{\partial^{2} R}{\partial z \partial \bar{z}}(z)+\frac{\partial^{2} R}{\partial z \partial z}(z)=\frac{\partial^{2} R}{\partial x \partial z}(z)=O(y)=O(k)
$$


By (22)

$$
a=\frac{1}{2 \pi} k^{2}(2 y)^{-2}+O(k)=2 \pi \phi^{\prime}(0)^{2}+O(k)
$$

and

$$
\begin{aligned}
|b|^{2} & =k^{2} \phi^{\prime \prime}(0)^{2}+O\left(k^{3}\right), \\
-\operatorname{Re} b & =\frac{1}{4 \pi} k^{2} \operatorname{Re}\left(f^{\prime \prime} / f^{\prime}\right)^{\prime}(0)+\delta k y \phi^{\prime \prime \prime}(0)+O\left(k^{3}\right) \\
& =\frac{1}{4 \pi} k^{2}\left(\operatorname{Re}\left(f^{\prime \prime} / f^{\prime}\right)^{\prime}(0)-\phi^{\prime \prime \prime}(0) / \phi^{\prime}(0)\right)+O\left(k^{3}\right) .
\end{aligned}
$$

Let $\beta=\left(\phi^{\prime \prime} / \phi^{\prime}\right)^{\prime}(0)-\operatorname{Re}\left(f^{\prime \prime} / f^{\prime}\right)^{\prime}(0)$. By (2) and (14) $\beta>0$. Since

$$
\begin{aligned}
|b|^{2}-2 a \operatorname{Re} b & =k^{2} \phi^{\prime}(0)^{2}\left[\left(\left(\phi^{\prime \prime} / \phi^{\prime}\right)(0)\right)^{2}+\operatorname{Re}\left(f^{\prime \prime} / f^{\prime}\right)^{\prime}(0)-\left(\phi^{\prime \prime \prime} / \phi^{\prime}\right)(0)\right]+O\left(k^{3}\right) \\
& =-\beta k^{2} \phi^{\prime}(0)^{2}+O\left(k^{3}\right),
\end{aligned}
$$

it follows that $|a-b|=a-(\beta / 4 \pi) k^{2}+O\left(k^{3}\right)$. (23) now follows from (25), proving the lemma.

To complete the proof of the theorem, we put the second derivative matrix $D^{2} W(z, \zeta)$ in block form

$$
D^{2} W(z, \zeta)=\left[\begin{array}{ll}
M & L \\
L^{t} & N
\end{array}\right]
$$

where $N=D_{\zeta}^{2} W(z, \zeta)$ is positive definite for $k$ sufficiently small since $D^{2} W_{0}(\widetilde{\zeta})$ is positive definite. By (10) all terms in $L$ are $O(k)$, but since the smaller eigenvalue of $M$ is $O\left(k^{2}\right)$, this is not yet enough information to conclude that $D^{2} W(z, \zeta)$ is positive. Let $\delta$ be a positive lower bound for the eigenvalues of $N$ for $k$ sufficiently small. For vectors $u \in R^{2}$, $v \in R^{2 p}$, let $u=u_{1}+u_{2}$ where $u_{j}$ is an eigenvector of $M$ for $\lambda_{j}$. Then

$$
\left|2 u_{1} L v^{t}\right| \leq C k\left\|u_{1}\right\|\|v\| \leq \frac{1}{4}\left(\lambda_{1}\left\|u_{1}\right\|^{2}+\delta\|v\|^{2}\right) \leq \frac{1}{4}\left(u_{1} M u_{1}^{t}+v N v^{t}\right)
$$

for $k$ sufficiently small. To obtain a similar inequality for $u_{2}$ we first note that since $\operatorname{Im} \phi_{1}(z, \zeta)=\psi_{1}(z, \zeta)$ is constant (and independent of $\zeta$ ) for real $z$, it follows that $\operatorname{Im}\left(\partial \phi_{1} / \partial \zeta_{j}\right)^{\prime}(z, \zeta)=0$ for real $z ;$ so $\operatorname{Im}\left(\partial \phi_{1} / \partial \zeta_{j}\right)^{\prime}(z, \zeta)=O(y)=O(k)$ for $(z, \zeta)=$ $(z(k), \zeta(k))$. Therefore by $(10)$

$$
\frac{\partial^{2} W}{\partial x \partial \zeta_{j}}=2 \operatorname{Re} \frac{\partial^{2} W}{\partial z \partial \zeta_{j}}=-\delta k \operatorname{Im}\left(\frac{\partial \phi_{1}}{\partial \zeta_{j}}\right)^{\prime}(z, \zeta)=O\left(k^{2}\right) .
$$

Also from (24) and (23) we find that an eigenvector of $M$ for $\lambda_{2}$ is $(a-\operatorname{Re} b+|a-b|, \operatorname{Im} b)$. It follows from (26), (27), and (28) that the $y$-component of a unit eigenvector $e$ for $\lambda_{2}$ is $O(k)$. Therefore

$$
e \cdot\left(\frac{\partial^{2} W}{\partial x \partial \zeta_{j}}, \frac{\partial^{2} W}{\partial y \partial \zeta_{j}}\right)=O\left(k^{2}\right), \quad 1 \leq j \leq p
$$

and hence

$$
\begin{aligned}
\left|2 u_{2} L v^{t}\right| & \leq C k^{2}\left\|u_{2}\right\|\|v\| \leq C_{1} k \sqrt{\lambda_{2}} \sqrt{\delta}\left\|u_{2}\right\|\|v\| \\
& \leq \frac{1}{4}\left(\lambda_{2}\left\|u_{2}\right\|^{2}+\delta\|v\|^{2}\right) \leq \frac{1}{4}\left(u_{2} M u_{2}^{t}+v N v^{t}\right)
\end{aligned}
$$


for $k$ sufficiently small. It follows that $\left|2 u L v^{t}\right| \leq \frac{1}{2}\left(u M u^{t}+v N v^{t}\right)$ and consequently that $D^{2} W(\zeta, z)$ is positive definite for $k$ sufficiently small.

3. Applications. Several situations where the above theorem can be applied will be discussed briefly. The proof in the previous section does not provide any information on how large $k$ may be taken and still have a stable configuration. This depends heavily on the geometry. In some cases it can be shown numerically as in [7] that the locus of possible stable vortex locations can be extended to fairly large values of $k$.

The computed examples given below have been obtained by the following procedure: The conformal map of the flow domain to a canonical domain (a half-plane, interior or exterior of the unit disk, or annulus) is computed and all terms in the formula for $\partial W / \partial z$ computed from (10) and known formulas for $g$ and $H$ on the canonical domain. A numerical routine based on Powell's method (a compromise between Newton's method and steepest descent) is used to determine the roots of the system of nonlinear equations (7). For this problem the routine frequently diverges unless a reasonable initial guess for the vortex locations is provided. The asymptotic formula (22) can be used as an initial guess for small $k$. By incrementing $k$, vortex locations are obtained for larger values of $k$. Stability is determined by computing the eigenvalues of the second derivative matrix numerically. The procedure can be continued until saddle points of $W$ are encountered on the locus. Subsections (c) and (d) refer to recent computations requiring a doubly connected analog of the Schwarz-Christoffel transformation and which appear in a joint work with Alan Elcrat and Chenglie $\mathrm{Hu}[2]$.

Before turning to specific examples, we state three simple, but rather general principles that indicate situations in which vortices can be added to a steady flow configuration.

(1) If for some bounded component $\Gamma$ of the boundary, a flow has no stagnation point on $\Gamma$, then a stable stationary vortex can be added near some point of $\Gamma$.

(2) If $P$ is a convex corner of the boundary and $P$ is not a stagnation point of the flow, then a stable stationary vortex can be added near $P$.

(3) If a domain $D$ has two consecutive nonconvex corners and a flow in $D$ has no stagnation point between them, then a stable stationary vortex can be added near some boundary point between the two nonconvex corners.

The above statements are immediate consequences of Theorem 2 and the following observations: For (1), $q$ must have a minimum at some point on the compact set $\Gamma$; for (2), $q=0$ at $P$; and for (3), $q$ is large at the two nonconvex corners and hence must have a minimum between them.

To be absolutely correct, in applying Theorem 2 in both cases (1) and (3) above one needs to know that the minimum is nondegenerate. Nondegeneracy is certainly the generic case, but there are situations when it does not hold, as noted in two examples below. So in this regard (1) and (3) should be taken as guidelines rather than absolutely rigorous statements. Regarding these statements as guidelines, it is also noted that the term "corner" in (2) and (3) can in practice be extended to include rounded corners as well as strict corners. In each of the specific examples given below nondegeneracy of the minimum can be determined either analytically or numerically. 


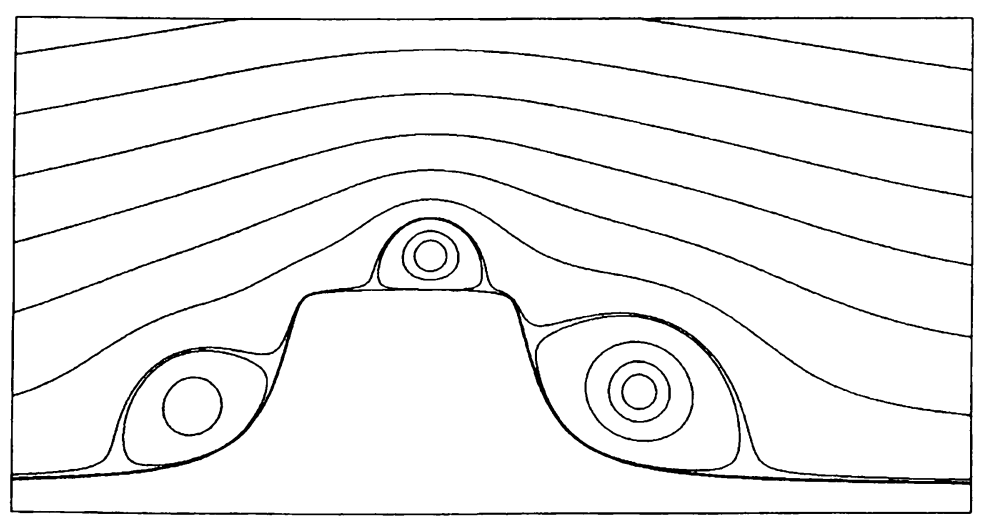

FIG. 1. Flow past an obstacle with three stable vortices: a vortex of strength 3.1 between the two rounded nonconvex corners, and vortices of strengths 2.0 and 3.0 in the rounded convex corners

As a simple example consider potential flow in the domain shown in Fig. 1. The fluid speed has three relative minima on the boundary, one in each of the two rounded convex corners and one between the two consecutive nonconvex corners. Figure 1 shows a computed configuration of three stable stationary vortices in the given domain.

Most of the examples that follow make use of (1) or (3) above. Examples of corner vortices have been given in [7].

a) Bounded simply connected domains. For any bounded simply connected domain the Routh function for one vortex has a minimum at some interior point $\zeta_{0}$; so there is a flow with a vortex at $\zeta_{0}([4])$. The resulting flow has no stagnation point on the boundary $\Gamma$; so by principle (1) it should be possible to add a second stable, stationary vortex near a point $P$ on $\Gamma$ where the speed has a minimum. If $\Gamma$ is a circle this minimum is degenerate because of rotational symmetry; so the result does not apply. (Positions for a second stationary vortex can be found in a circular disk, but they are unstable because of the symmetry.)

As a simple example of a noncircular domain, computations were done when $D$ is the image of the unit disk under the conformal map $z \mapsto z+a z^{2},|a|<1 / 2$. (The boundary $\Gamma$ is a limaçon.) Although the theorem only implies the existence of a second vortex for small values of the circulation, by using the incremental continuation procedure described at the beginning of the section it was found that for any choice of $k_{1}$ and $k_{2}$ of opposite sign, there is a flow in $D$ with stable vortices of strengths $k_{1}$ and $k_{2}$. By scaling we may assume $k_{1}=-1$. When $k_{2}$ is small the first vortex is near $\zeta_{0}$ and the second near $P$. As $k_{2}$ increases to infinity, the second vortex moves toward $\zeta_{0}$ and the first to the reflection $P^{\prime}$ of $P$ in the axis of symmetry. Figure 2 shows the configuration when $k_{2}=0.8(a=0.2)$. It is conjectured that the statement in italics is true for a general noncircular bounded simply connected domain.

For the domain $D$ considered in the previous paragraph the speed for the flow with a single vortex at $\zeta_{0}$ has two local minima on the boundary. Hence there are flows with three stable vortices in $D$. A computed example is given in Fig. 3 . In contrast to the 


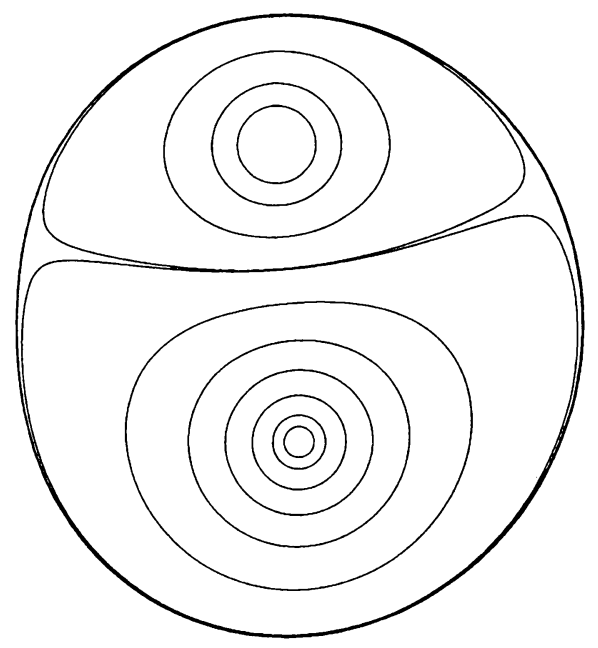

FIG. 2. A configuration of two stable vortices in the image $D$ of the unit disk under tie map $z \mapsto z+0.2 z^{2}$. The values of $k$ are -1.0 and 0.8 . A configuration of two stable vortices exists for any choice of $k_{1}$ and $k_{2}$ of opposite sign.

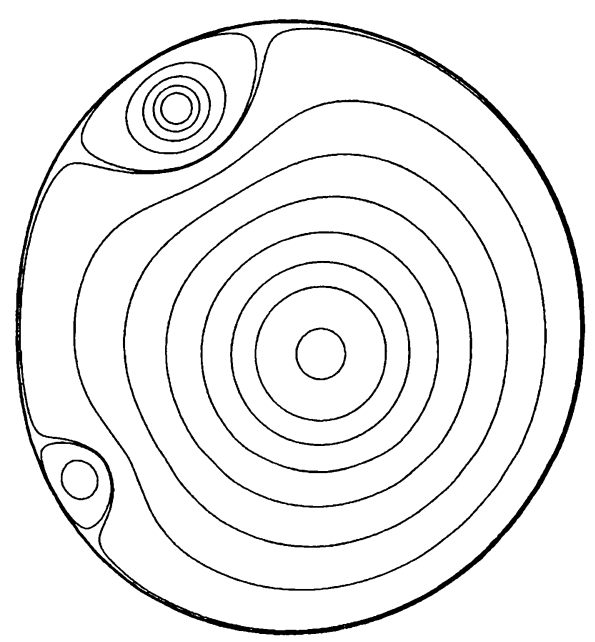

FIG. 3. A configuration of three stable vortices in the domain $D$ of Fig. 2. The values of $k$ are $-1.0,0.36$, and 0.2 .

case of two vortices where $k_{2}$ could be increased without bound, in this case it is not possible to increase either $k_{2}$ or $k_{3}$ (keeping the other fixed) much beyond the values indicated for Fig. 3. Between consecutive stagnation points for the flow in Fig. 3 the speed has no local minima; so it is reasonable to conjecture that the maximum number of stable vortices for steady flow in this domain is three.

As an example of a domain supporting a greater number of stationary vortices, consider the image of the unit disk under the map $z \mapsto(z / a)+\frac{1}{6}(z / a)^{6}, a=1.15$, shown in 


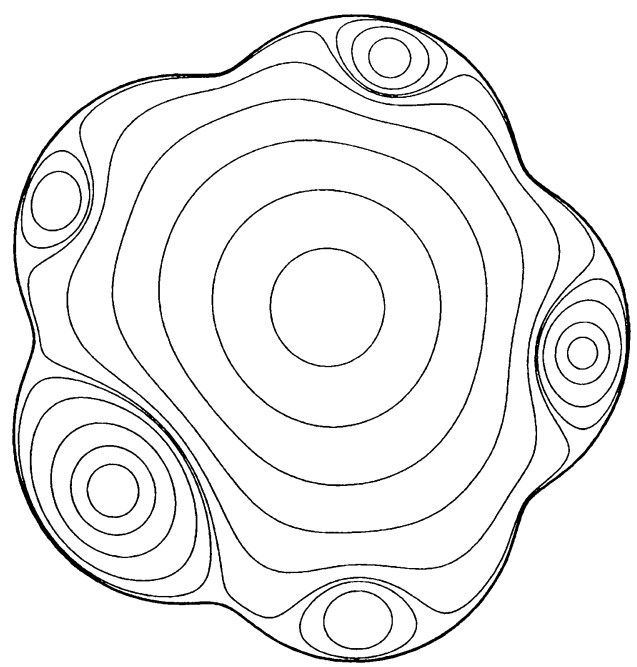

FIG. 4. A configuration of six stable vortices in the image of the unit disk under the map $z \mapsto(z / a)+\frac{1}{6}(z / a)^{6}, a=1.15$. The values of $k$ are $-1.0,0.5,0.15,0.25,0.3$, and 0.15 .

Fig. 4. The speed of the one vortex initial flow for this domain has five local minima. Figure 4 shows a flow with six stable stationary vortices of various strengths.

b) Annular domains, exterior domains. Let $\Gamma_{1}$ and $\Gamma_{2}$ be nonintersecting simple closed curves with $\Gamma_{2}$ inside $\Gamma_{1}, D$ the annular domain between $\Gamma_{1}$ and $\Gamma_{2}$. Consider irrotational flow in $D$ with circulation $K$ about $\Gamma_{2}$ (the flow with stream function $\frac{K}{2 \pi} \log |f(z)|$, where $f$ is a conformal map from $D$ to a circular annulus centered at 0 .) The flow has no stagnation point on either $\Gamma_{1}$ or $\Gamma_{2}$; so by principle (1), generally there will be flows with two stable vortices in $D$, one attached to $\Gamma_{1}$, the other to $\Gamma_{2}$. Figure 5 gives a computed example where the boundaries are nonconcentric circles. (The case of two concentric circles is an exception: the speed of irrotational flow is constant on both boundary components and any added vortex will be unstable because of the symmetry.) If the inner boundary is a convex polygon, then by principle (3) a vortex can be added along each side of the polygon.

Similar remarks apply to irrotational flow with circulation in a domain exterior to a simple closed curve $\Gamma$. One may include the possibility of a nonzero uniform velocity at infinity, in which case there is no stagnation point on $\Gamma$ if the circulation $K$ about $\Gamma$ is sufficiently large. It is possible to add at least one vortex near $\Gamma$ in such cases and $n$ vortices if $\Gamma$ is a convex $n$-gon.

c) Vortices attached to an airfoil. Principle (3) can be similarly applied to flow past an airfoil (the leading and trailing edges are nonconvex, possibly rounded, corners). The flow domain $D$ may either be unbounded externally or bounded externally by one or two curves parallel to the $x$-axis at infinity (channel flow). A uniform velocity at infinity is assumed. If the angle of attack is not too large, then for a certain range of values of the circulation $K$ about the airfoil, potential flow in $D$ will have two stagnation points 


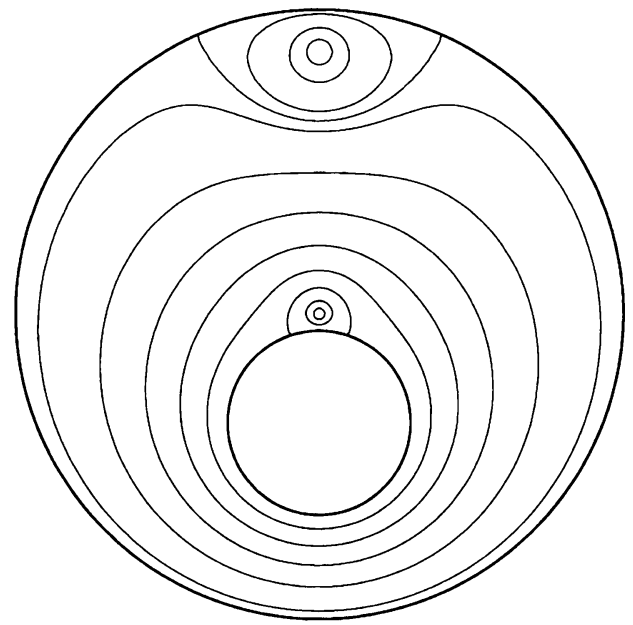

FIG. 5. In a typical annular region it is possible to have stable vortices attached to both the inner and outer boundaries. For the configuration shown, the vortices have strength $\pm \frac{1}{2} K$ where $K$ is the circulation about the inner boundary.

on the lower side of the airfoil and no stagnation point on the upper side. Applying principle (3), a stationary vortex can be added along the upper side of the airfoil. If $K$ is held fixed the stagnation points on the lower part of the airfoil are closer together after a vortex is added. It is more interesting to consider the one-parameter family of solutions obtained by varying $K$ so that $K+k$ remains constant, where $k$ is the strength of the vortex. Then the rear stagnation point moves closer to the trailing edge as $k$ increases. If the angle of attack is positive but not too large, then $k$ can be increased to obtain a flow with a stable vortex above the airfoil and satisfying the Kutta condition at the trailing edge.

These flows have aerodynamic significance because the attached vortex provides added lift to the airfoil. When there are no external boundaries such vortices have been studied both analytically and numerically by Saffman and Sheffield [9] when the airfoil is a flat plate and by Huang and Chow [5] in the case of Joukowski airfoils. Some computed examples when the airfoil is in a channel are given in [2].

If the circulation $K$ about the airfoil is so large that there is no stagnation point on the airfoil, then (3) implies that vortices may be added along both the upper and lower sides of the airfoil. Figure 6 (see p. 566) gives a computed example of such a configuration. (To avoid infinite velocity, both the leading and trailing edges have been rounded in this example.)

REMARK. A few caveats should be made about applying principles (2) and (3) at rounded corners. First, for an actual convex corner, applying the main result successively implies that for any $N$ there exist flows with a sequence of $N$ alternately positive and negative vortices descending into the corner at $P$. But if the corner is rounded, then there will be a bound on the number of vortices that can be added in the corner, that bound depending in part on the curvature of the boundary near the corner. For example, 


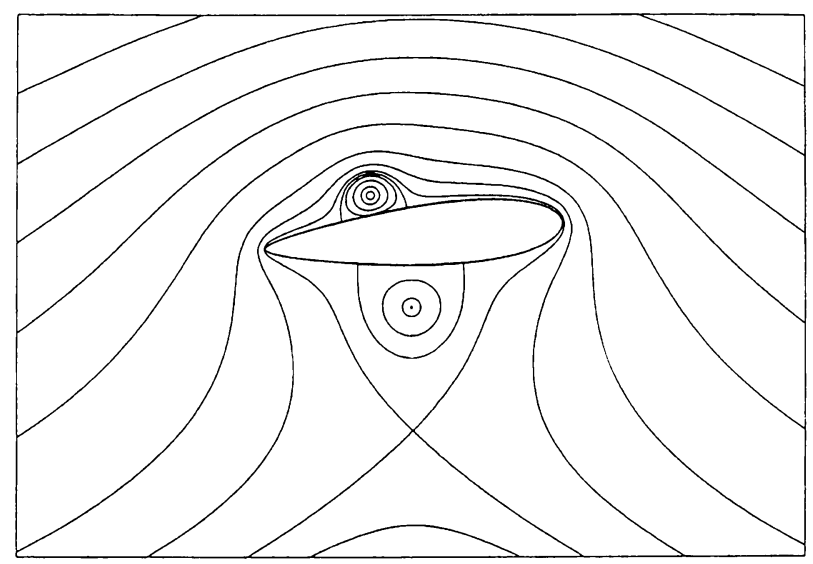

FIG. 6. Flow past an airfoil with two attached vortices. The circulation around the boundary of the airfoil is $K=4.5$. For the vortex above the airfoil $k=4.0$; for the vortex below the airfoil $k=1.0$.

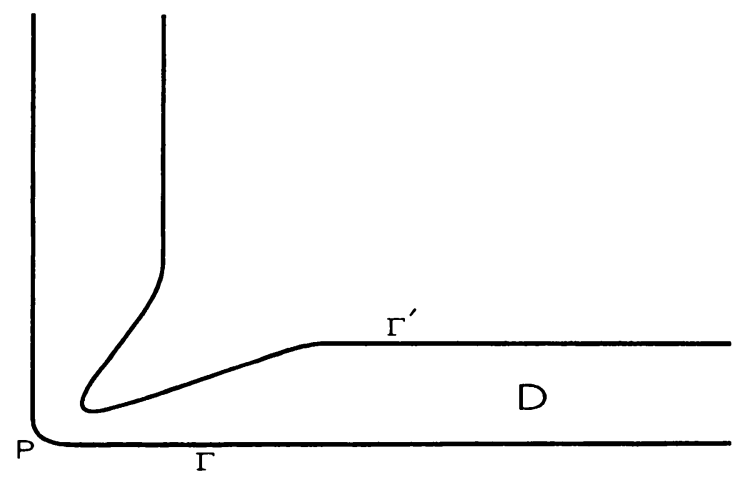

FIG. 7. For potential flow in the channel $D$, the existence of a relative minimum for the speed near the rounded corner $P$ is dependent on how narrow the channel is near $D$, not simply on the curvature of $\Gamma$ near $P$.

for the flow in Fig. 1, a secondary vortex cannot be added in either corner. Second, even when adding a single vortex to potential flow it should be noted that the hypotheses of Theorem 2 are not simply local conditions on the boundary: the speed of potential flow depends on non-local properties. As a simple illustration, consider potential flow in a channel $D$ between the curves $\Gamma$ and $\Gamma^{\prime}$ in Fig. 7. Depending on the narrowness of the channel at $P$ the speed of potential flow in $D$ may or may not have a local minimum near the rounded corner $P$. This example also shows that a criterion based solely on the curvature of the boundary at $P$ will not suffice to imply that a vortex can be added near $P$. Similar examples can be constructed to show that principle (3) is not always valid when the corners are rounded. 

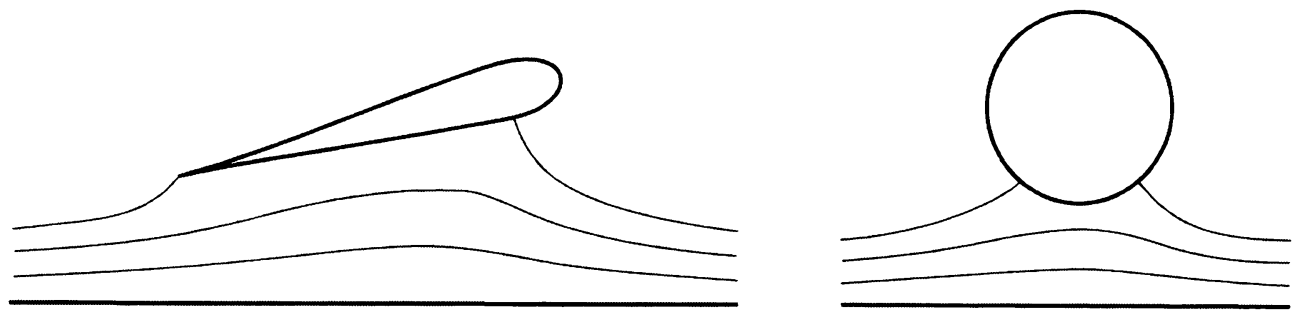

FIG. 8. Two examples of flows past an interior obstacle in a halfplane for which condition $C$ is satisfied. A stable vortex may be added near some point on the lower (straight line) boundary.

As a final example of the main theorem's applicability, a somewhat different argument will be used to show that there can be a steady vortex attached to the ground below a low-flying aircraft (the vortex moving with the same speed as the aircraft).

d) Ground (or wall) vortices for flow past an obstacle. Consider potential flow in a half-plane (or channel with parallel walls) past an interior obstacle. Assume that the $x$-axis $\Gamma$ is the boundary of the half-plane with the obstacle in the upper half-plane, that the velocity at infinity is $(-1,0)$ and that there is no stagnation point on $\Gamma$. If there is an $x_{0}$ such that the speed $q<1$ at $\left(x_{0}, 0\right)$, then there will be some point $(x, 0)$ where $q$ restricted to $\Gamma$ is a minimum and hence there will be stable vortex locations near $(x, 0)$. There is an easily visualized geometric condition which implies that $q<1$ at some $x_{0}$. Say that condition $C$ is satisfied at $x_{0}$ if there is a neighborhood $N$ of $\left(x_{0}, 0\right)$ such that each streamline passing through $N$ is higher in $N$ than it is in the far field. Condition $C$ is equivalent to $y>\psi(x, y)$ on $N$, where $\psi$ is the stream function. Thus condition $C$ implies $q=\partial \psi / \partial y<1$ at $\left(x_{0}, 0\right)$. Condition $C$ is satisfied for slender obstacles with positive angle of attack (see Fig. 8a) and for more arbitrary obstacles if there is sufficient circulation about the obstacle (Fig. 8b). A computed example of a flow with a vortex attached to the wall of a channel when the obstacle is a flat plate is given in [2].

Acknowledgments. The author was partially supported by NSF-EPSCOR grant OSR-9255223.

\section{REFERENCES}

[1] M. S. Berger and L. E. Fraenkel, Nonlinear desingularization in certain free-boundary problems, Commun. Math. Phys. 77, 149-172 (1980)

[2] A. R. Elcrat, C. Hu, and K. G. Miller, Equilibrium configurations of point vortices for channel flow past interior obstacles, European J. Mech. B Fluids 16, 277-292 (1997)

[3] A. R. Elcrat and K. G. Miller, Rearrangements in steady multiple vortex flows, Comm. Partial Differential Equations 20, 1481-1490 (1995)

[4] B. Gustafsson, On the motion of a vortex in two-dimensional flow of an ideal fluid in simply and multiply connected domains, Research Report, Dept. of Mathematics, Royal Institute of Technology, Stockholm, 1979

[5] M. K. Huang and C. Y. Chow, Trapping a free vortex by Joukowski airfoils, AIAA Journal 20, 292-298 (1982)

[6] C. C. Lin, On the Motion of Vortices in Two Dimensions, Univ. Toronto Appl. Math. Series, no. 5, Toronto Univ. Press, 1943 
[7] K. G. Miller, Stationary corner vortex configurations, Z. angew. Math. Phys. 47, 39-56 (1996)

[8] P. G. Saffman, Vortex Dynamics, Cambridge University Press, Cambridge, 1992

[9] P. G. Saffman and J. Sheffield, Flow over a wing with an attached free vortex, Studies in Applied Math. 57, 107-117 (1977)

[10] B. Turkington, On steady vortex flow in two dimensions, I, Comm. Partial Differential Equations 8, 999-1030 (1983)

[11] B. Turkington and A. Eydeland, An iterative method for computing steady vortex flow systems, Proceedings of the Workshop on Mathematical Aspects of Vortex Dynamics (R. Caflisch, ed.), SIAM, Philadelphia, 1989 , pp. 80-87

[12] Y. H. Wan, Desingularization of systems of point vortices, Phys. D 32, 277-295 (1988) 